\title{
Comparison of Analog and Digital Self-Powered Systems in Multimodal Vibration Suppression
}

\author{
Shigeru Shimose, ${ }^{1}$ Kanjuro Makihara, ${ }^{2}$ and Junjiro Onoda ${ }^{1}$ \\ ${ }^{1}$ Institute of Space and Astronautical Science (ISAS), Japan Aerospace Exploration Agency (JAXA), 3-1-1 Yoshinodai, \\ Chuo-Ward, Sagamihara, Kanagawa 252-5210, Japan \\ ${ }^{2}$ Department of Aerospace Engineering, Tohoku University, 6-6-01 Aoba, Aramaki, Aoba-Ward, Sendai 980-8579, Japan
}

Correspondence should be addressed to Shigeru Shimose, shimose.shigeru@jaxa.jp

Received 14 December 2011; Accepted 15 January 2012

Academic Editor: Ma Jan

Copyright (C) 2012 Shigeru Shimose et al. This is an open access article distributed under the Creative Commons Attribution License, which permits unrestricted use, distribution, and reproduction in any medium, provided the original work is properly cited.

This paper compares our analog and digital self-powered systems for vibration suppression, and shows experimental results of multimodal vibration suppression for both self-powered systems. The experimental results are evaluated in light of the damping performance and adaptability under various vibrational conditions. We demonstrate various examples of our innovative vibration suppression method, called "digital self-powered." Proper status switching of an electric circuit made up of an inductor and a selective switch connected to a piezoelectric transducer attenuates the vibrations. The control logic calculation and the switching events are performed with a digital microprocessor that is driven by the electrical energy converted from the mechanical vibration energy. Therefore, this vibration suppression system runs without any external power supply. The self-powering feature makes this suppression method useful in various applications. To realize an ideal vibration suppression system that is both self-powered and effective in suppressing multimode vibration, sophisticated control logic is implemented in the digital microprocessor. We demonstrate that our digital self-powered system can reduce the vibrational displacements of a randomly excited multimodal structure, by as much as $35.5 \%$.

\section{Introduction}

Vibration control methods are roughly categorized into two groups, that is, active and passive methods [1-4]. Active vibration control methods usually yield high-performance vibration suppression $[1,2]$. However, active control systems may become unstable if the control is improperly designed. In addition, active vibration control systems need an external energy supply to suppress vibrations. On the other hand, passive vibration control methods use energy dissipative mechanisms such as dampers, frictional devices, and electric resistors $[3,4]$. Because passive approaches do not need an energy supply they are always stable. Passive methods are easier to implement in actual systems than are the more complicated active methods, because they do not need controllers, sensors, or filters. However, in most cases, passive systems do not provide a satisfactory vibration suppression performance. In general, the majority of passive systems suppress vibrations well only in expected situations such as those regarding natural frequency and temperature. Typical examples of less robustness of frequency alternation are mechanically tuned mass dampers and electrical dynamic vibration absorbers. There are research papers that compare the performance of semiactive and passive systems $[5,6]$.

We found a new approach to resolve the dilemma between active and passive vibration controls in terms of robustness and suppression performance. The semiactive method is one of the practical answers both to reduce the disadvantages of active systems and to maintain the advantages of passive systems. Many studies of semiactive vibration control methods have been published, and they often use piezoelectric transducers for vibration suppression [7-14]. Some researchers have studied a semiactive approach to suppress vibration by using piezoelectric transducers with switchable circuits [10-14]. Richard et al. [10], Corr and Clark [11], and Onoda et al. [12] proposed the use of an inductive circuit. Depending on the circuit components and 
the switching strategies, this approach is called SSDI, LRswitching, RL-shunt, and others. By shunting the transducer in short time, the counter electromotive force that is generated by the inductor and the restored electric charge due to the piezoelectric effect can invert the polarity of the charge stored in it. Accordingly, the stored electrical energy in the transducer is recycled rather than immediately dissipated, and is often called an energy-recycling semiactive method [12]. The energy-recycling semiactive vibration control does not require any external power supply to suppress vibration. The systems without the need for external energy are quite useful for various applications $[13,14]$ in energy shortage systems and those that require energy saving, such as large space structures, artificial satellites, and isolated lunar bases, all of which are vulnerable to facing long periods without sunlight.

So far, we have developed two types of self-powered vibration controllers: analog [15] and digital [16]. Both types of the controllers do not require external energy. In this paper, we compare our analog [15] and digital self-powered [16] systems for multimodal vibration suppression from the viewpoints of damping performance and adaptability to various vibrational conditions.

\section{Problem Statements and Research Objectives}

The energy-recycling semiactive vibration control does not require any external energy to suppress vibrations. However, switches, switching controllers, and other electric devices contained in the energy-recycling semiactive vibration control devices consume electrical energy. To avoid external power supply, we did not make use of the above devices in the vibration suppression system. Thus, an analog selfpowered system using the semiactive technique $[15,17,18]$ has been proposed. The "analog self-powered" system is the simplest type among self-powered vibration controllers. This system requires neither an external power supply nor an external control authority. However, analog circuit systems are very awkward in practice and not at all programmable. If the parameters need changing due to system alternations, analog systems cannot deal with such requests. Therefore, to solve the awkwardness and inflexibility issues associated with analog self-powered systems, we investigated "digital self-powered" systems for sophisticated vibration control. Single degree of freedom (SDOF) structural vibration suppression experiments using our "digital self-powered" system indicated that the vibration magnitude dramatically reduced by as much as $79.7 \%$ [16]. Moreover, we have attempted to apply our "digital self-powered" system to multimodal structural vibration suppression.

Here, we revealed the experimental performance effectiveness of our proposed analog and digital self-powered systems for multimodal structural vibration suppression control. First, we describe the energy-recycling, semiactive mechanism (switching method for semiactive vibration suppression and the equations of motion for a multimodal structure). Next, an explanation of our self-powered systems is presented. Finally, experimental results are demonstrated in

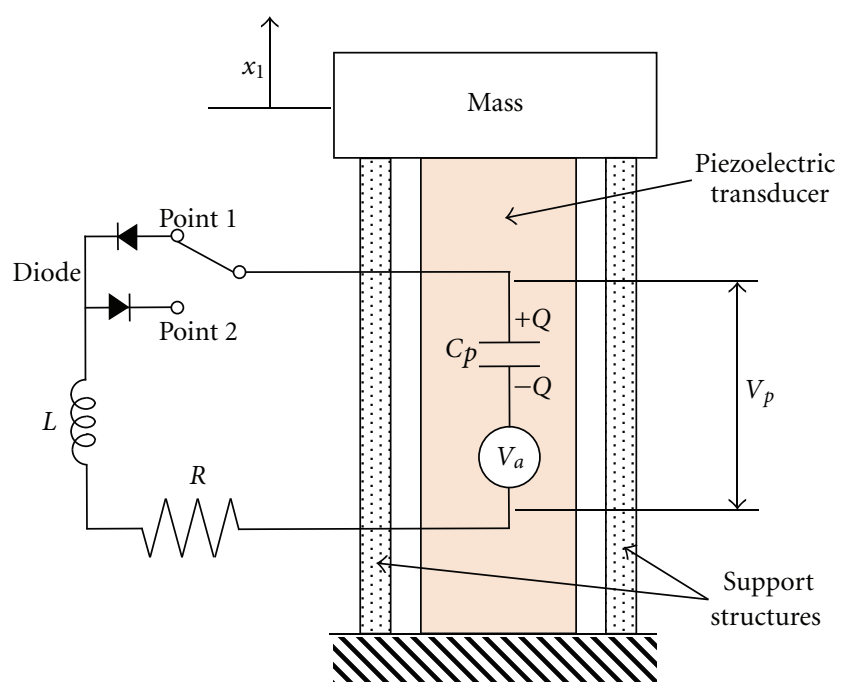

FIGURE 1: SDOF system with switching circuit for energy-recycling.

terms of damping performance under multimodal vibration excitation.

\section{Theoretical Analysis}

3.1. Switching Method for Semiactive Vibration Suppression. This section shows the basic idea of the energy-recycling semiactive mechanism that has been reported in [10-12]. However, for a better understanding, a brief explanation is presented using the simple SDOF system shown in Figure 1. The system is composed of a mass, support structures, a piezoelectric transducer, and a switchable inductive circuit. The linear characteristic of the piezoelectric transducer [19] is written as

$$
\begin{gathered}
f_{p}=k_{p} x_{1}-b_{p} Q, \\
V_{p}=-b_{p} x_{1}+\frac{Q}{C_{p}},
\end{gathered}
$$

where $f_{p}$ is the applied force, $x_{1}$ is the elongation of the piezoelectric transducer, $Q$ is the electric charge stored in the transducer, $V_{p}$ is the piezoelectric voltage across two electric terminals, $C_{p}$ is the constant-elongation piezoelectric capacitance, and $b_{p}$ is the piezoelectric constant. The piezoelectric transducer is modeled with a voltage generator $V_{a}$ and a capacitor $C_{p}$. Here, let us assume that the SDOF system with natural frequency $\omega$ is vibrating as

$$
x_{1}=A \sin (\omega t) \text {. }
$$

Active controls can freely change the value of $Q$ for vibration control. The optimal control charge (defined as $Q_{T}$ ) is derived from active control schemes. For instance, the direct velocity feedback control [20] stipulates the control input as

$$
Q_{T}=-F \frac{d x_{1}}{d t}
$$


where $F$ is the feedback gain.

In contrast to the abovementioned active controls, our switching system (Figure 1) has only a selective switch to change its electrical state. This is the semiactive method that does not have an external power source and cannot change the value of $Q$ as expected. Our switching logic is that the selective switch is connected to point 1 or 2 to make the polarity of $Q$ the same as that of $Q_{T}$. The switching logic $[12]$ is written as follows

when $Q_{T}<0$, change the switch into point 1, when $Q_{T} \geq 0$, change the switch into point 2 .

We assume that $Q$ is zero at $t=0$. Equations (1) and (2) indicate that $V_{p}$ is negative when $x_{1}$ is positive and $Q$ is zero. While the switch is positioned at point 1 , the diode at point 1 prevents the electric current from flowing in the circuit. Consequently $x_{1}$ and $Q$ are zero, and $V_{p}$ is $-b_{p} A$ just before turning the switch at $t=\pi /(2 \omega)$. After the switch is connected to point 2 at $t=\pi /(2 \omega)$, the electric current flows in the inductive circuit. The governing equation of the electric circuit while the current flows is expressed as

$$
L \ddot{Q}+R \dot{Q}+\frac{Q}{C_{p}}=b_{p} x_{1},
$$

where $\omega \sqrt{L C_{p}} \ll 1$ according to study [12]. We also assume that the electric current stops flowing in a much shorter time than the period of the mechanical vibration. Thus, $x_{1}$ can be thought to remain equal to $A$ during current flow. The values of $Q$ and $V_{p}$ just after the current stops flowing are

$$
\begin{gathered}
Q=C_{p} b_{p} A\left(1+e^{-\zeta \pi}\right), \quad V_{p}=b_{p} A e^{-\zeta \pi}, \\
\zeta \equiv \frac{R}{2} \sqrt{\frac{C_{p}}{L}} .
\end{gathered}
$$

$x_{1}$ and $V_{p}$ and $Q$ remain constant, because the diodes prevent the current from flowing. At $t=(3 \pi) /(2 \omega), x_{1}$ changes to $-A$ and $V_{p}$ becomes

$$
V_{p}=b_{p} A e^{-\zeta \pi}+2 b_{p} A
$$

At this moment, the switch is turned to point 1 according to the control logic (5), and the current starts to flow. After the current flow stops due to the diode at point $1, Q$ and $V_{p}$ are

$$
\begin{gathered}
Q=-C_{p} b_{p} A\left(1+2 e^{-\zeta \pi}+e^{-2 \zeta \pi}\right), \\
V_{p}=-b_{p} A\left(2 e^{-\zeta \pi}+e^{-2 \zeta \pi}\right) .
\end{gathered}
$$

After many repetitions of switching, the values of $Q$ and peak voltage converge to

$$
\begin{gathered}
|Q|=C_{p} b_{p} A \frac{1+\gamma}{1-\gamma}, \quad\left|V_{\text {Peak }}\right|=b_{p} A\left(\frac{1+\gamma}{1-\gamma}+1\right), \\
\gamma \equiv e^{-\zeta \pi}
\end{gathered}
$$

Similarly, the dissipated energy $E_{D}$ for one cycle is

$$
E_{D}=4 C_{p} b_{p}^{2} A^{2} \frac{1+\gamma}{1-\gamma} .
$$

The parameter $\gamma$ is in direct correlation to the dissipated energy and is called the voltage inversion coefficient. It is defined in [13] as the ratio of voltages after and before the voltage inversion.

\section{Equation of Motion of the Multimodal Structure and Controller}

For deriving the generalized equations, let us consider an l-DOF structure with piezoelectric transducers. The equation of motion for the structure with piezoelectric transducers [12] can be written as

$$
\mathbf{M} \ddot{\vec{u}}+\mathbf{D} \dot{\vec{u}}+\mathbf{K} \vec{u}=\mathbf{B}_{p} \vec{Q}+\vec{w} .
$$

To express (2) in vector-matrix form, we transform the scalar equation to

$$
\vec{V}_{p}=-\mathbf{B}_{p}^{T} \vec{u}+\mathbf{C}_{p}^{-1} \vec{Q} .
$$

In modal coordinates, the equation of motion is expressed as

$$
\ddot{\vec{\xi}}+\Xi \dot{\vec{\xi}}+\Omega \vec{\xi}=\Phi^{T} \mathbf{B}_{p} \vec{Q}+\Phi^{T} \vec{w},
$$

and the voltage equation is written as

$$
\vec{V}_{p}=-\mathbf{B}_{p}^{T} \Phi \vec{\xi}+\mathbf{C}_{p}^{-1} \vec{Q}
$$

where

$$
\begin{gathered}
\Phi \equiv\left[\phi_{1}, \phi_{2}, \ldots, \phi_{l}\right], \quad \Omega \equiv \operatorname{diagonal}\left[\omega_{k}^{2}\right], \\
\Xi \equiv \operatorname{diagonal}\left[2 \zeta \omega_{k}\right], \quad(1 \leq k \leq l) .
\end{gathered}
$$

Then, (14) and (15) can be transformed into

$$
\begin{aligned}
\dot{\vec{z}} & =\mathbf{A} \vec{z}+\mathbf{B} \vec{Q}+\mathbf{E} \vec{w}, \\
\vec{V}_{p} & =\mathbf{C} \vec{z}+\mathbf{D} \vec{Q},
\end{aligned}
$$

where

$$
\begin{gathered}
\vec{z} \equiv\left[\begin{array}{cc}
\vec{\xi} & \overrightarrow{\vec{\xi}} \\
&
\end{array}\right]^{T}, \quad \mathbf{A} \equiv\left[\begin{array}{cc}
0 & \mathrm{I} \\
-\Omega & -\Xi
\end{array}\right], \quad \mathbf{B} \equiv\left[\begin{array}{cc}
0 \\
\Phi^{T} & \mathbf{B}_{p}
\end{array}\right], \\
\mathbf{C} \equiv\left[\begin{array}{lll}
-\mathbf{B}_{p}^{T} & \Phi & 0
\end{array}\right], \quad \mathbf{D} \equiv \mathrm{C}_{p}^{-1}, \quad \mathbf{E} \equiv\left[\begin{array}{c}
0 \\
\Phi^{T}
\end{array}\right] .
\end{gathered}
$$

If $\vec{Q}$ in (18) is regarded as an active control input, the linear quadratic regulator (LQR) control theory [21] specifies that the performance index

$$
J \equiv \int_{0}^{\infty}\left(\vec{z}^{T} \mathbf{W}_{1} \vec{z}+\vec{Q}^{T} \mathbf{W}_{2} \vec{Q}\right) d t
$$




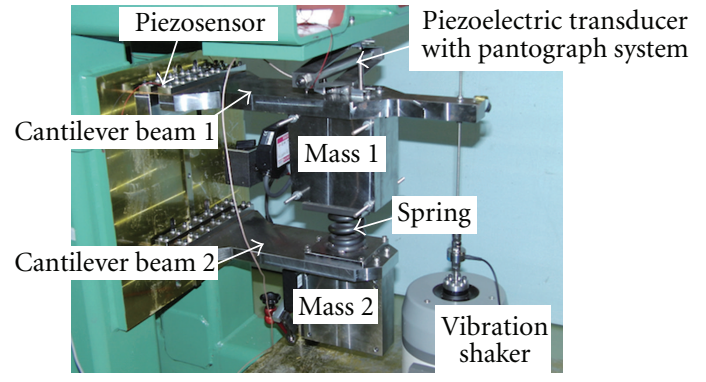

Figure 2: Photo of mechanical part in 2-DOF system.

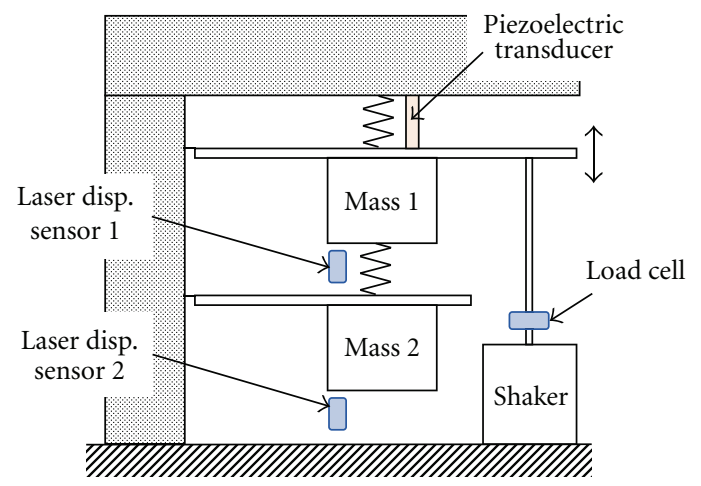

FIGURE 3: Schematic of mechanical configuration of 2-DOF system.

is minimized by

$$
\vec{Q}=\vec{Q}_{T} \equiv-\mathbf{F} \vec{z}
$$

where

$$
\mathbf{F} \equiv \mathbf{W}_{2}^{-1} \mathbf{B}^{T} \mathbf{P}_{1}
$$

Here, $\mathbf{W}_{1}$ and $\mathbf{W}_{2}$ are weighting matrices, and $\mathbf{P}_{1}$ is a positive solution of

$$
\mathbf{P}_{1} \mathbf{B} \mathbf{W}_{2}^{-1} \mathbf{B}^{T} \mathbf{P}_{1}-\mathbf{A}^{T} \mathbf{P}_{1}-\mathbf{P}_{1} \mathbf{A}-\mathbf{W}_{1}=0 .
$$

The vector $\vec{Q}_{T}$ is an evolved form of the simple scalar in (4). While $\vec{Q}_{T}$ can handle multiple transducer systems, the simple scalar form $Q_{T}$ can handle only single-transducer system.

\section{Experimental Configuration}

5.1. Mechanical Experimental System. A view of the mechanical experiment system is shown in Figures 2 and 3. The mechanical experimental system consists of two masses, a pantograph-type displacement-magnification mechanism, a piezoelectric transducer (PSt 1000/10/200-VS18, Piezomechanik $\mathrm{GmbH}$ ), piezoelectric sensors, two cantilevered beams, a vibration shaker, and an experimental platform. The displacement-magnification mechanism, attached to the upper beam and upper side of the platform, is used to amplify the displacement.
Figure 4 shows the electrical circuits of the self-powered system. The piezoelectric transducer is connected to the analog self-powered electrical device or the digital self-powered electric device. One piezoelectric sensor is attached to the base of the upper beam. Additional measuring instruments (a laser displacement sensor and load cell) are arranged around the system. These measuring instruments are used only for recording and not for the semiactive feedback control. For the purpose of effective analysis, an equivalent twoDOF spring-mass model is constructed for the experimental system. The natural frequencies of the first and second modes at constant electric charge are 20.3 and $36.6 \mathrm{~Hz}$, respectively. A detailed description of the experimental setup is given in our previous paper [16].

5.2. Electric Experimental System. The electrical circuit of the proposed analog self-powered system is shown in Figure 5. This circuit is designed with one energy-recycling section and two peak detection sections. The energy-recycling section has a piezoelectric transducer, inductors, and thyristors. The thyristors work as both a switch and a diode. The peak detection section finds the peak voltage of the piezoelectric transducer.

When the voltage across the piezoelectric transducer $V_{p}$ reaches the maximum and starts to decrease, the programmable unijunction transistor PUT1 is automatically turned on, and the charge stored in capacitor $\mathrm{C} 1$ flows through the thyristor SCR1 as its gate current. As a result, the thyristor is turned on, and the charge stored in the capacitor of the piezoelectric transducer $C_{p}$ flows through the inductor. Because the thyristor prevents the electric current from flowing in the opposite direction, this turning on of the thyristor is equivalent to turning the switch in Figure 1 to point 1 . Conversely, when the value of $V_{p}$ reaches the minimum and starts to increase, PUT2 is turned on, and subsequently, SCR2 is turned on. This action is equivalent to turning the switch in Figure 1 to point 2. As a result, this analog circuit turns the switch in Figure 1 to point 1 when $V_{p}$ is approximately the maximum, and turns the switch to point 2 when $V_{p}$ is approximately the minimum. This is equivalent to the switching logic proposed and studied by Richard et al. [10]. This is the simplest design of a self-powered vibration suppression system. However, it is not practical to realize the switching logic for handling a multiple input multiple output (MIMO) system.

Figure 6 shows a diagram of the energy flow and control stream of our digital self-powered system. The energyharvesting section, comprising four connected diodes, can supply the harvested energy to the digital processor. The energy harvester, with a storage capacitor $C_{S}$, is connected to the piezoelectric transducer to collect vibration energy. The circuit harvests electrical energy in a piezoelectric transducer attached to the vibrating structure, and it supplies energy to the energy harvester and sends the collected energy to the microprocessor. The piezoelectric sensor is attached to the structure and outputs a voltage that is proportional to the upper mass's displacement. It is connected to the analog/digital (A/D) port of the digital processor. All the necessary functions for switching control are built into the 


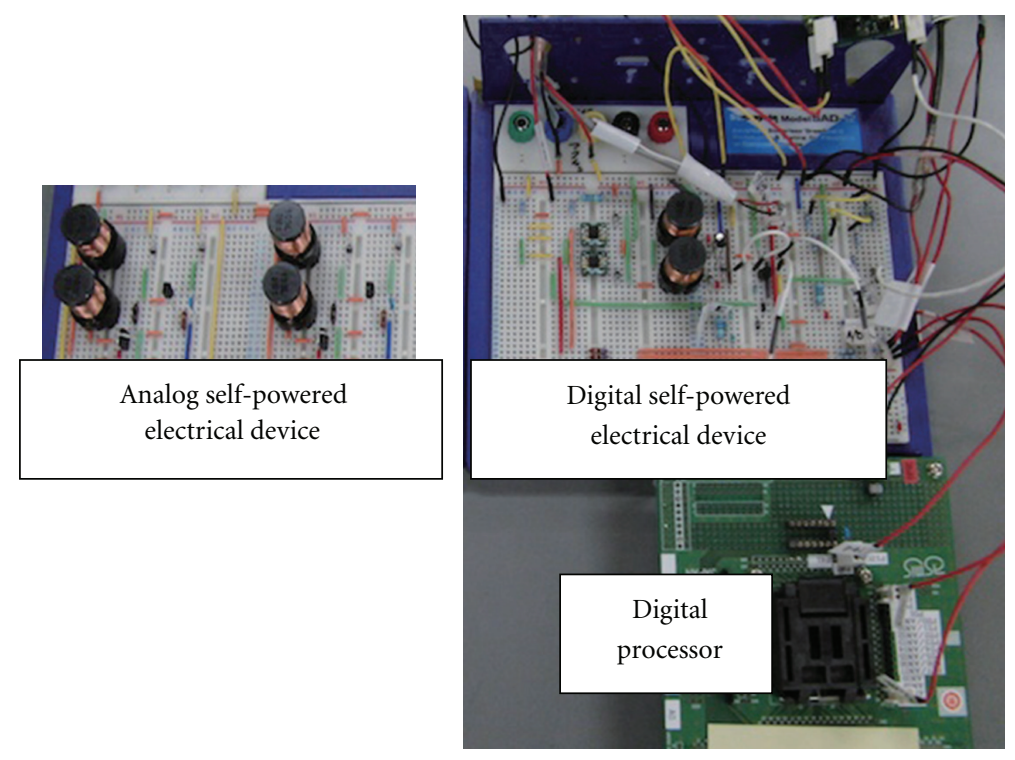

FIgURE 4: Photograph of electrical device in self-powered system.

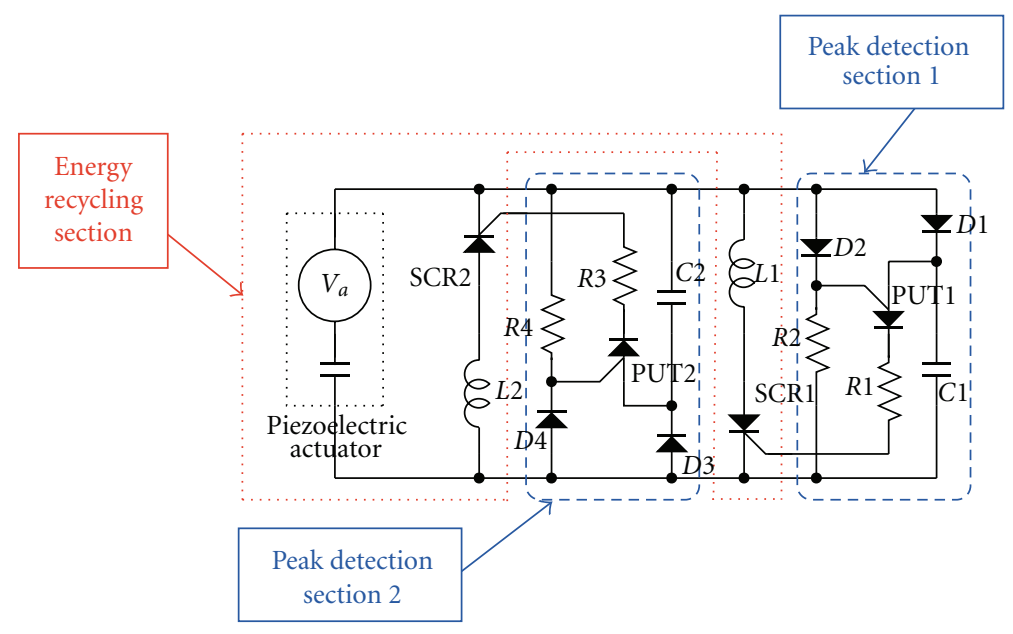

FIGURE 5: Analog self-powered control switching circuit.

digital processor. First, the digital processor activates using the power supplied energy-harvesting section. Second, it collects data pertaining to the structural vibrations from the piezoelectric sensor. Third, it calculates the modal estimation using the Kalman filter. Fourth, it calculates the switching criteria. Fifth, it activates the switch to inverse the voltage polarity of the piezoelectric transducer when the switching criteria polarity shifts. Finally, it monitors the transducer with the shifted voltage polarity that works a transducer to suppress structural vibrations. All of these steps are carried out at 0.5-millisecond intervals.

\section{Experimental Results}

This paper describes three types of vibration suppression experiments using our self-powered systems. Both self-powered systems presented in this paper utilize the same ontroller and observer designs described in our previous papers [15, 16].

6.1. Multimodal Sinusoidal Excitation. In this section, we carried out multimodal sinusoidal vibration suppression experiments using our analog and digital self-powered systems. A function generator creates an input voltage wave and sends it to the vibration shaker. The input wave for the vibration excitation is

$$
\alpha \sin \left(\omega_{1} t\right)+\frac{11}{7}(1-\alpha) \sin \left(\omega_{2} t\right)
$$

where $\omega_{1}$ and $\omega_{2}$ are the angular frequencies of the first and second modes, respectively. $\alpha$ is the modal combination parameter. The excitation with $\alpha=1$ means the sinusoidal excitation with frequency $\omega_{1}$ of the first mode, and the excitation with $\alpha=0$ means the sinusoidal excitation with 


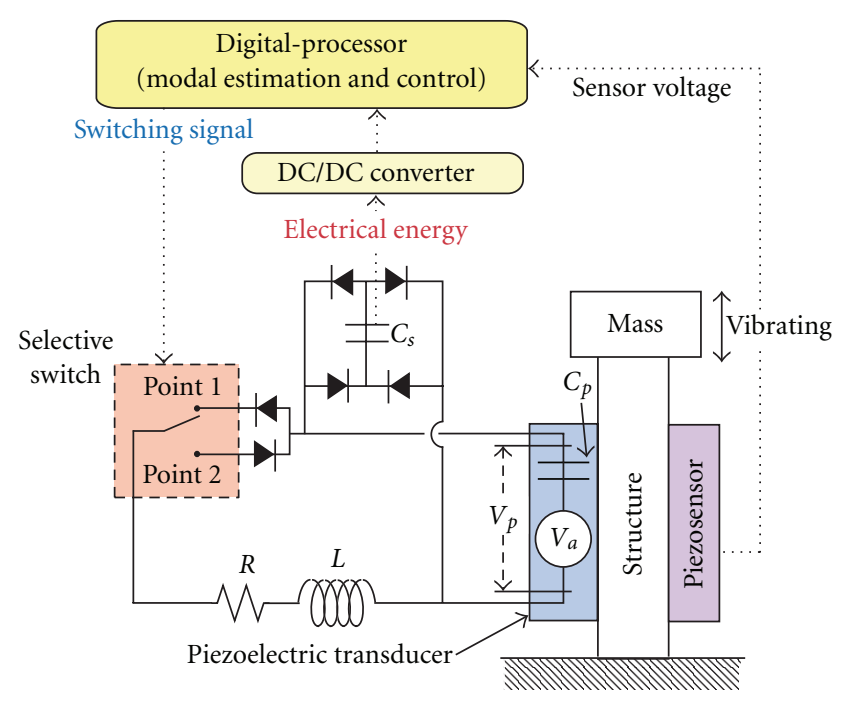

FIGURE 6: Control stream of digital self-powered vibration suppression system.

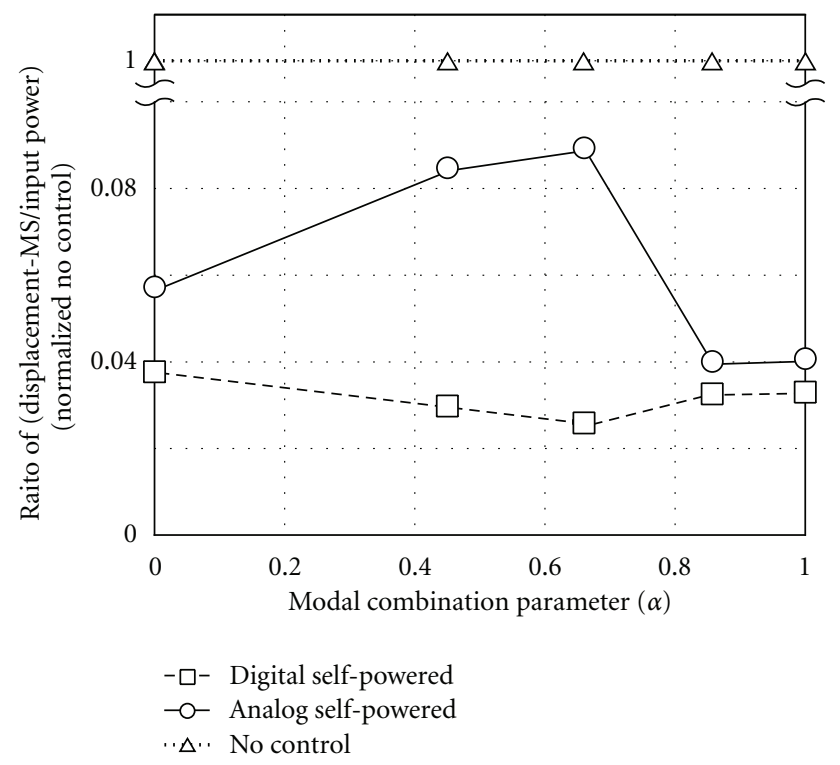

FIGURE 7: Comparison of displacement/input-power for various vibration suppression methods.

frequency $\omega_{2}$ of the second mode. The coefficient of the second mode was determined, so that the first and second modal vibrations had the same displacement level.

The comparison between the multimodal sinusoidal excitation experiments with the analog and digital selfpowered control systems is shown in Figure 7. The horizontal scale is the multimodal combination parameter, and the vertical scale is the ratio value of the mean square of the combined displacements of masses 1 and 2, divided by the input vibration power. These ratio values are normalized by the value of the no control case. This figure indicates that our digital self-powered control system can suppress all complicated vibrations. In contrast, our analog self-powered
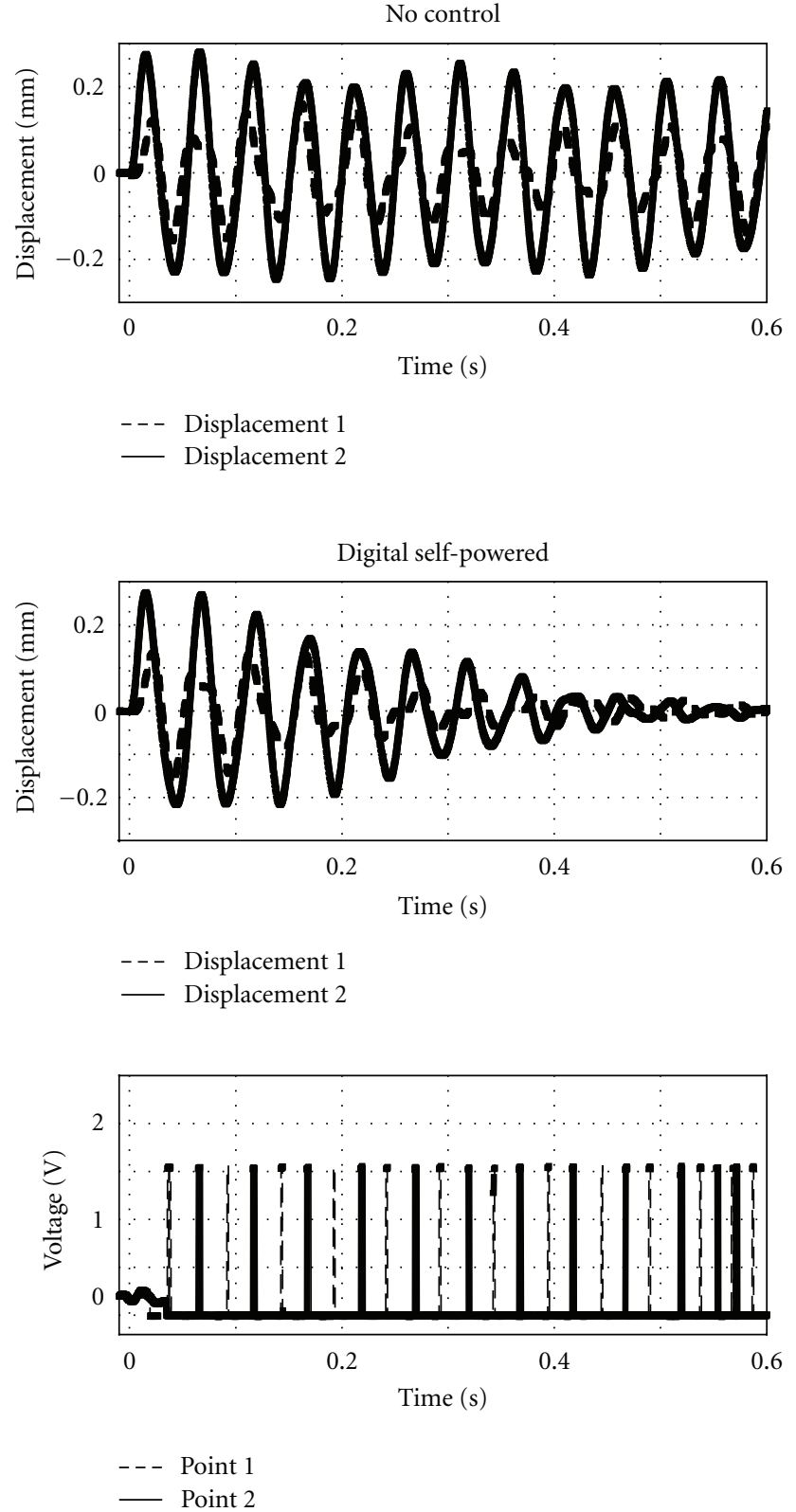

FIGURE 8: Experimental result of displacements under impulsive excitation with digital self-powered control.

control system is less effective when the combination of the two modes is around $0.4<\alpha<0.7$. This confirms that for suppressing complicated vibrations such as 2-mode vibrations, the digital self-powered system is better than the analog self-powered system.

6.2. Impulsive Excitation. We carried out impulse excitation experiments using our analog and digital self-powered systems. The impulse force was applied to mass 2 by an impulse hammer.

Figure 8 shows the displacements of the masses and the switching signal. The top of Figure 8 shows the displacements of masses 1 and 2 with no control. The middle of Figure 8 


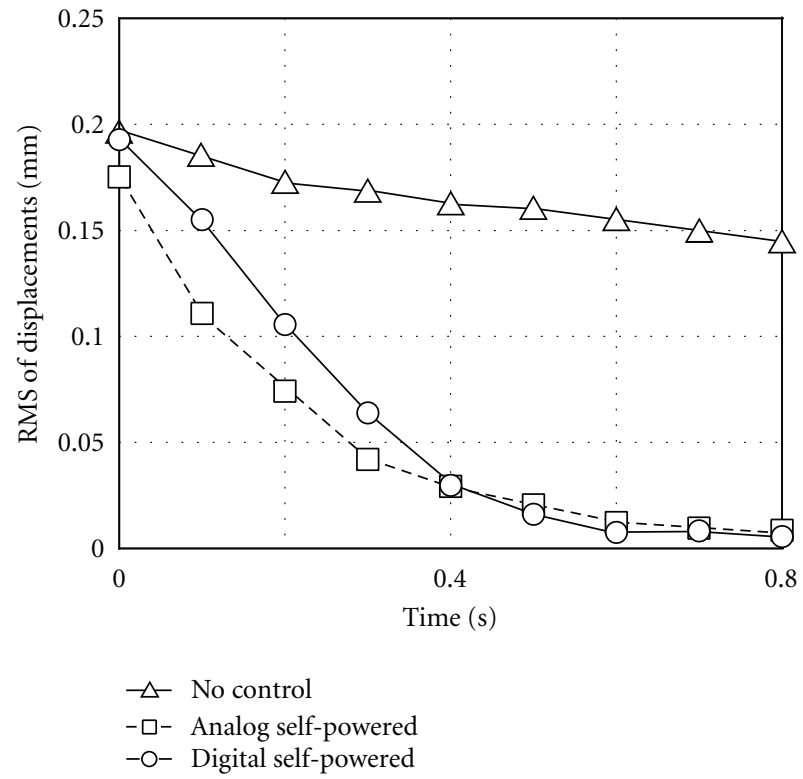

FIGURE 9: Comparison of RMS of displacements under impulsive excitation.
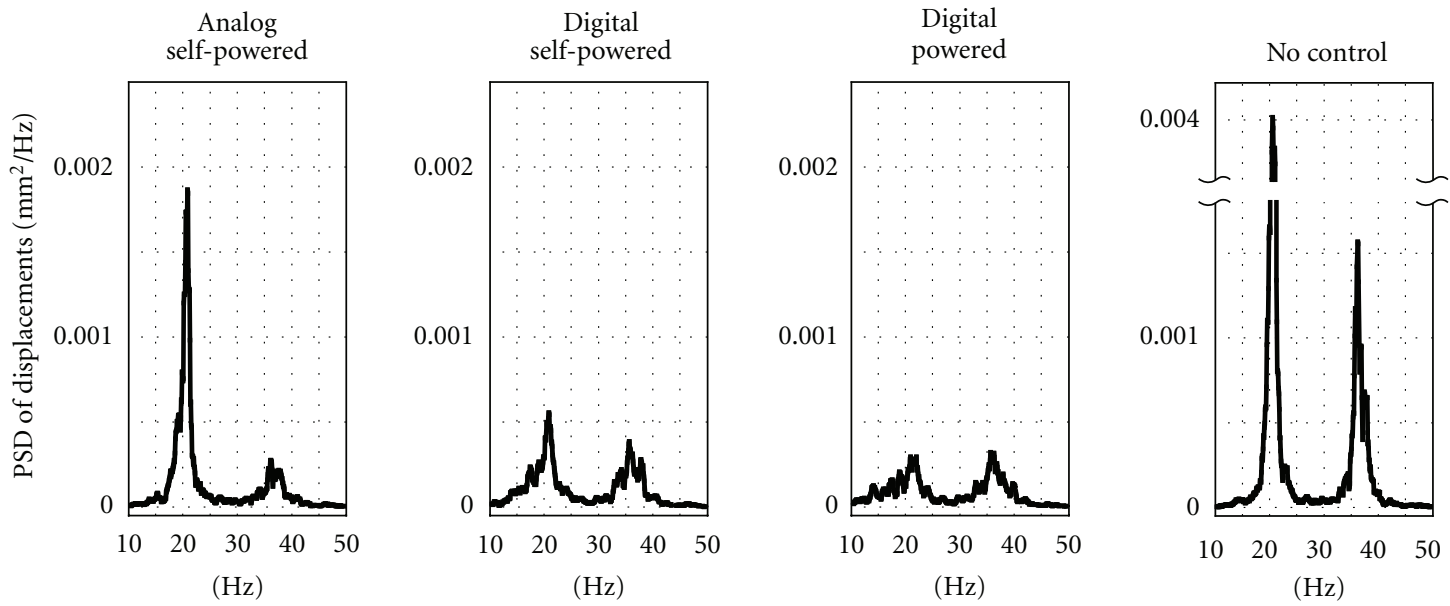

FIgURE 10: Comparison of PSD of displacements under random excitation.

shows the displacements of masses 1 and 2 with digital selfpowered control. The bottom of Figure 8 shows the signal voltage of the selective switch. Just after 0.035 seconds of input force, our digital self-powered system started the vibration suppression. At $0.5 \mathrm{~s}$, the magnitude of displacement 2 reduced from $0.215 \mathrm{~mm}$ to $0.021 \mathrm{~mm}$, a reduction of $90.4 \%$. These figures indicate that our digital self-powered system has a quick response and can therefore handle impulse dynamics.

Figure 9 shows the comparison between the impulse excitation experimental results of analog and digital selfpowered control systems. The horizontal scale is time, and the vertical scale is the root mean square of the combined displacements of masses 1 and 2. During the early period (from 0 to $0.3 \mathrm{~s}$ ), the analog self-powered system is more effective in vibration suppression than the digital one. This is quite natural because the digital self-powered system must only store some amount of electrical energy in the storage capacitor until the microprocessor enters the wake-up mode. However, overall, the suppression performances of both analog and digital controls are almost equivalent even for impulsive disturbances.

6.3. Random Excitation. In this section, we carried out random vibration suppression experiments using our analog and digital self-powered systems. A function generator creates an input voltage wave and sends it to the vibration shaker. The input wave for random vibration excitation is kept constant in the power spectral density (PSD) value, from 10 to $50 \mathrm{~Hz}$.

Figure 10 shows the comparison of the PSD displacements of masses 1 and 2 under random excitation. The 


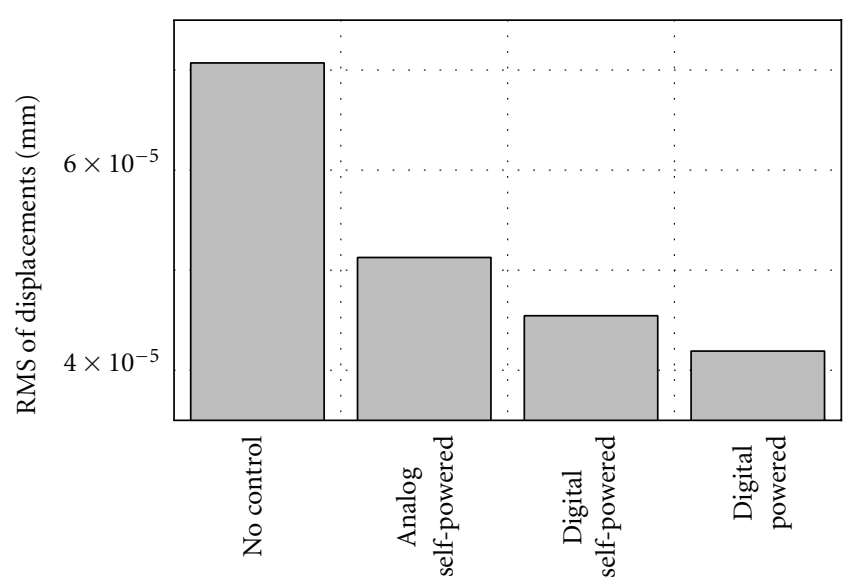

FIGURE 11: Comparison of RMS of displacements under random excitation.

horizontal scale is the frequency, and the vertical scale is the PSD of the displacement. The upper left side of Figure 10 shows the PSD value with analog self-powered control. The upper center of Figure 10 shows the PSD value with digital self-powered control. The upper right side of Figure 10 shows the PSD value with digital powered control. The lower part of Figure 10 shows the PSD value with no control. Unlike our self-powered systems, a digital powered system uses an external power supply for driving the digital processor. These figures indicated that the analog self-powered system could not suppress the first mode. However, the digital selfpowered system significantly reduced all modal amplitudes.

Figure 11 shows the comparison of the root mean square of the combined displacements of masses 1 and 2 . The experimental results demonstrate that the analog selfpowered system reduced the root mean square by $27.6 \%$, the digital self-powered system reduced it by $35.5 \%$, and the digital powered system reduced it by $40.7 \%$. The damping performance of the digital powered system was the most effective. This is quite natural because the digital powered system does not require an energy-harvesting section for driving the digital processor. Thus, these experimental results indicate that the lower the required harvested energy is, the better the damping performance of the digital self-powered system is.

\section{Conclusions}

This paper compared two types (i.e., analog and digital) of self-powered vibration suppression systems by using an energy recycling semiactive method with a piezoelectric transducer. These self-powered systems do not need an external power supply. The analog system comprises only analog electric devices whereas the digital system comprises an electric circuit with a programmable microprocessor.

We carried out several kinds of vibration suppression experiments using our proposed self-powered systems. These experiments clearly indicate that our self-powered systems yield a highly effective damping performance. Furthermore, we demonstrated that the digital self-powered system has significant advantages over the analog system in dealing with multimodal vibrations. These systems are useful for various applications where energy-saving or energy-shortage concerns exist, such as large-space structures, artificial satellites, and isolated lunar bases, which are all exposed to long periods of no sunlight.

\section{Acknowledgment}

This paper was supported by a Grant-in-Aid for Scientific Research (B) no. 20360386, from the Japan Society for Promotion of Science.

\section{References}

[1] M. Ahmadian and A. P. DeGuilio, "Recent advances in the use of piezoceramics for vibration suppression," Shock and Vibration Digest, vol. 33, no. 1, pp. 15-22, 2001.

[2] C. Niezrecki, D. Brei, S. Balakrishnan, and A. Moskalik, "Piezoelectric actuation: state of the art," Shock and Vibration Digest, vol. 33, no. 4, pp. 269-280, 2001.

[3] N. W. Hagood and A. von Flotow, "Damping of structural vibrations with piezoelectric materials and passive electrical networks," Journal of Sound and Vibration, vol. 146, no. 2, pp. 243-268, 1991.

[4] S. Y. Wu, "Piezoelectric shunts with a parallel R-L circuit for structural damping and vibration control," in Proceedings of the Smart Structures and Materials: Passive Damping and Isolation, vol. 2720 of Proceedings of SPIE, pp. 259-269, February 1996.

[5] D. Hrovat, P. Barak, and M. Rabins, "Semi-active versus passive of active tuned mass dampers for structural control," Journal of Engineering Mechanics, vol. 109, no. 3, pp. 691-705, 1983.

[6] J. Onoda, T. Sano, and K. Kamiyama, "Active, passive, and semiactive vibration suppression by stiffness variation," AIAA journal, vol. 30, no. 12, pp. 2922-2929, 1992.

[7] G. A. Lesieutre, "Vibration damping and control using shunted piezoelectric materials," Shock and Vibration Digest, vol. 30, no. 3, pp. 187-195, 1998.

[8] V. Giurgiutiu, "Review of smart-materials actuation solutions for aeroelastic and vibration control," Journal of Intelligent Material Systems and Structures, vol. 11, no. 7, pp. 525-544, 2000.

[9] W. W. Clark, "Vibration control with state-switched piezoelectric materials," Journal of Intelligent Material Systems and Structures, vol. 11, no. 4, pp. 263-271, 2000.

[10] C. Richard, D. Guyomar, D. Audigier, and H. Bassaler, "Enhanced semi passive damping using continuous switching of a piezoelectric device on an inductor," in Proceedings of the Smart Structures and Materials: Damping and Isolation, vol. 3989 of Proceedings of SPIE, pp. 288-299, March 2000.

[11] L. R. Corr and W. W. Clark, "Comparison of low-frequency piezoelectric switching shunt techniques for structural damping," Smart Materials and Structures, vol. 11, no. 3, pp. 370376, 2002.

[12] J. Onoda, K. Makihara, and K. Minesugi, "Energy-recycling semi-active method for vibration suppression with piezoelectric transducers," AIAA Journal, vol. 41, no. 4, pp. 711-719, 2003.

[13] S. Shimose, K. Makihara, K. Minesugi, and J. Onoda, "Assessment of electrical influence of multiple piezoelectric transducers' connection on actual satellite vibration suppression," 
Smart Materials Research, vol. 2011, Article ID 686289, 8 pages, 2011.

[14] K. Makihara, J. Onoda, and K. Minesugi, "Comprehensive assessment of semi-active vibration suppression including energy analysis," Transactions of ASME, Journal of Vibration and Acoustics, vol. 129, no. 1, pp. 84-93, 2007.

[15] J. Onoda, "Some advances in energy recycling semiactive vibration suppression," Advances in Science and Technology, vol. 56, pp. 345-354, 2008.

[16] K. Makihara, S. Takeuchi, S. Shimose, J. Onoda, and K. Minesugi, "Innovative digital self-powered autonomous system for multimodal vibration suppression," AIAA Journal. In press.

[17] D. Niederberger and M. Morari, "An autonomous shunt circuit for vibration damping," Smart Materials and Structures, vol. 15, no. 2, pp. 359-364, 2006.

[18] M. Lallart and D. Guyomar, "An optimized self-powered switching circuit for non-linear energy harvesting with low voltage output," Smart Materials and Structures, vol. 17, no. 3, Article ID 035030, 2008.

[19] B. Jaffe, W. R. Cook Jr., and H. Jaffe, Piezoelectric Ceramics, Academic Press, London, UK, 1971.

[20] M. J. Balas, "Direct velocity feedback control of large space structures," Journal of Guidance, Control, and Dynamics, vol. 2, no. 3, pp. 252-253, 1979.

[21] H. Kwakernaak and R. Sivan, Linear Optimal Control System, Wiley-Interscience, New York, NY, USA, 1972. 

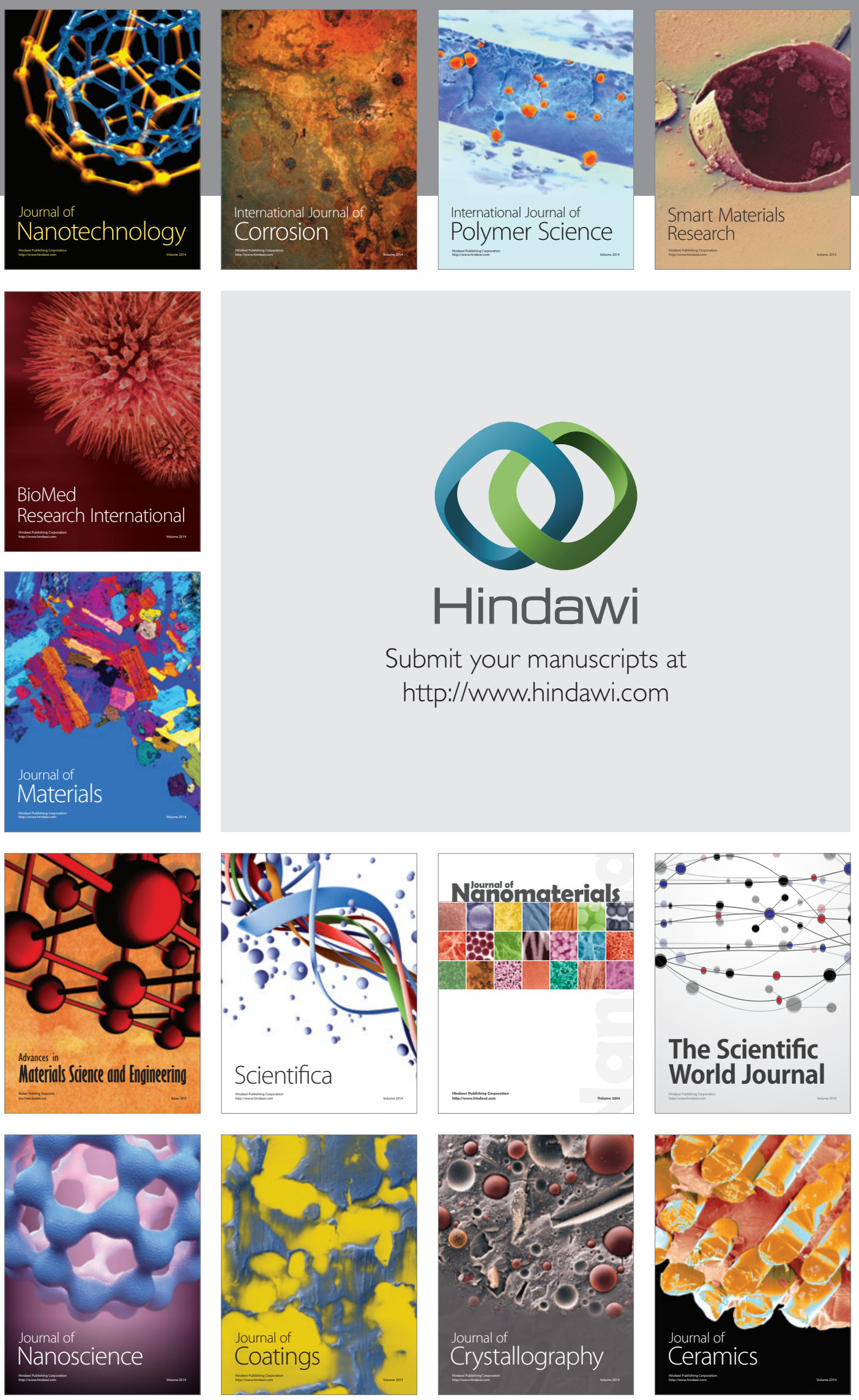

The Scientific World Journal

Submit your manuscripts at

http://www.hindawi.com

\section{World Journal}

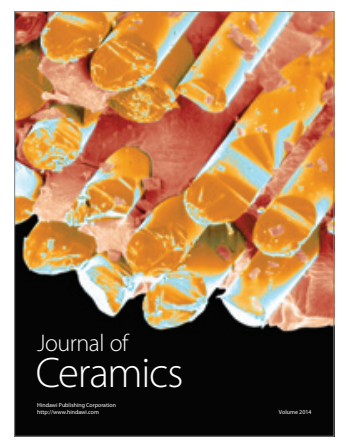

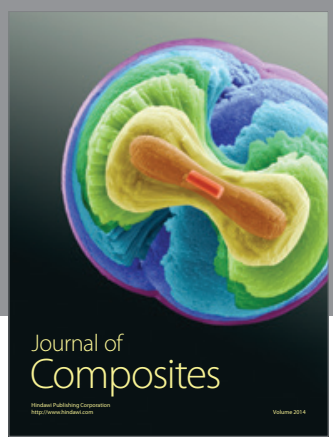
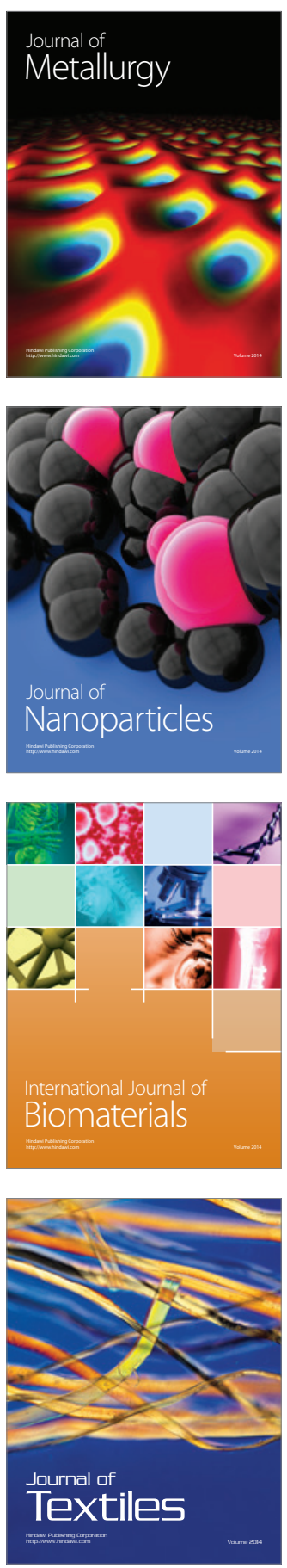